\title{
Perioperative genomics and neurologic outcome: we can't change who we are
}

\author{
Hilary P. Grocott, MD
}

Published online: 29 May 2009

(c) Canadian Anesthesiologists' Society 2009

Adverse cerebral outcomes have been described in most perioperative settings; however, for quite some time, cardiac surgery has been the poster child for cognitive dysfunction as well as incrementally severe complications, such as delirium, encephalopathy and stroke. ${ }^{1,2}$ Not that these complications do not occur in other operative settings, ${ }^{3}$ but with the incidence of post-cardiac surgery complications being generally higher than in other settings, cardiac surgery patients are an easily identifiable group to study in detail. Understanding the factors that influence perioperative cerebral outcome has never been more relevant, as not only are patients presenting for surgery at an increasingly older age (i.e., consistent with global demographic changes) but they frequently have multiple co-morbidities (many of which are risk factors for neurologic injury). Despite changes in the management of coronary artery disease (CAD) that have lead to a gradual decline in these procedures over the past 5-10 years, coronary artery bypass graft surgery (CABG) remains one of the most commonly performed operations. Furthermore, a recent large-scale study identified that the optimal management of multivessel CAD was with CABG, as opposed to percutaneous intervention. ${ }^{4}$ This may lead to a resurgence of CABG surgery with a further increase in the overall number of patients manifesting neurologic dysfunction.

Much has been written about the risk factors for adverse neurologic outcome. ${ }^{5,6}$ Although many factors are modifiable, such as the way to approach ascending aortic atheromatous disease, ${ }^{7}$ many are not, which is clearly the

H. P. Grocott, MD (ه)

Departments of Anesthesia and Surgery, University of Manitoba, CR3008-369 Tache Avenue, Winnipeg, MB R2H 2A6, Canada e-mail: hgrocott@sbgh.mb.ca case regarding one's genetic composition. Several recent trials have highlighted how our genetic milieu either increases the predisposition for neurologic injury or impairs the ability to recover once that injury has occurred. ${ }^{8-13}$ Despite pointing to identifiable yet sometimes divergent genetic risk factors, each of these studies has distinct strengths and weaknesses. However, regardless of reports of significant findings that provide provocative scientific insights, the study and understanding of perioperative genomics is in its relative infancy. Indeed, there are several thematic deficiencies present in most of the reported studies. The first and foremost shortcoming relates to the relatively small size of most trials. Although some of the trials are larger than the majority of other perioperative clinical trials, they are smaller than needed to avoid the problems of poor statistical power, multiple comparisons, and the seemingly ever-changing bioinformatics field. However, advancements in analytical techniques with expanded capability and accuracy have facilitated gathering the needed genetic information in a more cost-effective manner, making it possible to study larger populations.

The incredible complexity of our genetic code ${ }^{14}$ presents an expanding challenge to our ability to fully understand the effects of genetic variation. Variations in genetic make-up have been described by the concept of single nucleotide polymorphisms (SNPs), where individual nucleotides (i.e., base pairs) differ within specific genes, thus producing various alleles. The limitations of analytical techniques have restricted the number of SNPs that can reasonably be interrogated. It has also led us to examine specific and pre-determined, though biologically plausible, targets. However, these a priori defined SNPs may completely miss the more important and as yet unknown variants. As a result, whole-genotype analysis, although suffering its own set of limitations, has improved the 
ability to look beyond the limitations of a priori variants. ${ }^{15}$ On the other hand, it has also been criticized for spawning diffuse and non-hypothesis-driven fishing expeditions.

With this background in mind, there are several significant and intriguing studies, both cardiac and non-cardiac, that have added to our understanding of perioperative neurologic outcome and genomics. ${ }^{13,16}$ The first investigations of perioperative genetic variation and cerebral outcome (now almost 15 years old) focused on single gene variants. In a small study $(n=65)$ reported by Tardiff $e t$ al., one of the first SNPs examined was the apolipoprotein (APOE) variant where the $\varepsilon 4$ allele was associated with cognitive dysfunction (POCD). ${ }^{17}$ The reasons for examining this variant grew from a body of literature that outlined associations of APOE with various adverse cerebral outcomes, such as Alzheimer's disease, subarachnoid hemorrhage, closed head injury, and others. ${ }^{18,19}$ However, as attempts were made with various corroborative studies, significant questions were raised as to how robust the relationship was between this $\varepsilon 4$ variant and POCD. Indeed, Steed et al. failed to demonstrate a similar relationship in the setting of cardiac surgery, and Gaynor et al. later found an opposite result in the pediatric population, with the $\varepsilon 2$ (not the $\varepsilon 4$ ) variant being associated with adverse cerebral outcome. ${ }^{9,11}$ Although Leung et al. showed a potential APOE link to POCD in non-cardiac surgery, ${ }^{20}$ a relationship that was also seen by Heyer et al. in carotid endarterectomy patients, ${ }^{21}$ it is clear that there are equally and potentially more important variants.

The next SNP that was examined in cardiac surgery was the polymorphism to the platelet integrin receptor $\left(\mathrm{PL}^{\mathrm{A} 2}\right)$. Mathew et al. described the influence of this variation of the GPIIIa constituent of the GPIIb/IIIa platelet integrin receptor (one that was investigated because of a known association with an increased incidence of acute coronary syndromes) ${ }^{22}$ on outcome in patients as determined using the mini-mental status examination (MMSE). ${ }^{10}$ Although this study $(n=70)$ demonstrated that the $\mathrm{PL}^{\mathrm{A} 2}$ positive patients had lower MMSE results, the relative insensitivity of the MMSE results, as well as the small study size, somewhat limited their findings.

Although there were inherent limitations in these early trials, they did lay important groundwork for further, more elaborate studies linking genetics and adverse cerebral outcomes after cardiac surgery. More recently, Grocott et al. reported the results of a large study $(n=2,104)$ investigating links between post-cardiac surgery stroke and up to 26 different SNPs. This study not only included a much larger number of SNPs than previously investigated, but importantly, it also considered SNP to SNP interactions. Indeed, the interaction of $\mathrm{C}$-reactive protein (CRP) and interleukin-6 (IL-6) SNPs was associated with a significantly increased risk of stroke. The combination of the two minor alleles of CRP (3'UTR $1846 \mathrm{C} / \mathrm{T})$ and IL-6 $(-174 \mathrm{G} / \mathrm{C})$ imposed a threefold increase in the risk of stroke (odds ratio [OR], 3.3; 95\% confidence interval [CI], 1.4$8.1 ; P=0.0023) .{ }^{23}$ The incidence of stroke in a population with the genotype was $3.09 \%$ compared with an incidence of $0.95 \%$ in a population without. This study highlighted a potential mechanistic pathway for perioperative stroke; inflammatory superseded thrombosis-related genes vis-àvis the relationship to stroke after cardiac surgery.

Where imaging modalities and focused neurologic examinations can be used to identify deficits, stroke is, in many ways, easier to study in the perioperative setting than POCD. The POCD phenotype is a much more difficult endpoint to investigate, requiring cumbersome yet detailed assessments of cognitive function that are difficult to accomplish on the large scale that is necessary to fully understand the influences of genetic variation. Despite these limitations, Mathew et al. described an elegant study ( $n=$ 443) outlining a novel effect of P-selectin (SELP) and CRP SNPs. ${ }^{8}$ They described that the minor alleles (minor because of their less frequent occurrence) of CRP 1059G/C (OR 0.37, 95\% CI 0.16-0.78; $P=0.013$ ) and SELP 1087G/A (OR $0.51,95 \%$ CI $0.30-0.85 ; P=0.011)$ were actually associated with a reduction in overall cognitive deficits. The absolute risk reduction in the incidence of POCD was $20.6 \%$ for those with the CRP 1059C allele and $15.2 \%$ for those carrying the SELP 1087A allele. Furthermore, perioperative serum CRP levels and platelet activation markers were also significantly lower in patients with a copy of these minor alleles, supporting the biologic plausibility of reduced inflammation and platelet activation for the observed allelic associations of better cognitive outcome.

Without question, the field of perioperative genomics is dynamic and evolving. Just as our early understanding of APOE and its relationship to brain injury has been replaced with an increasingly complex but mechanistically plausible understanding, we can only expect that further refined understanding of genetic influences will continue in tandem with the investigative process.

Where do we go from here? First, because of the statistical and technical limitations outlined previously, any significant findings (those already reported and those still to come) need corroboration. It is difficult to know whether the already identified genetic risk factors will hold up to further scrutiny, yet this phase is essential before progressing to the required next step-targeted genetic risk stratification. Until these important intermediate step(s) are more completely understood, an even further step beyond understanding risk is the development of various therapeutic strategies based on one's genetic composition. Many building blocks have been laid down thus far; however, the foundation remains incomplete. Ultimately, work in perioperative genomics should lead to an increased 
understanding of pathophysiology, risk assessment, and, eventually, attempts to mitigate the effects of perioperative neurologic injury with preventative and therapeutic intervention.

\section{Génomique périopératoire et devenir neurologique: nous ne pouvons changer qui nous sommes}

Les complications cérébrales ont été décrites dans la plupart des contextes périopératoires; toutefois, depuis un certain temps déjà, la chirurgie cardiaque est le symbole du dysfonctionnement cognitif ainsi que des complications de plus en plus graves telles que le delirium, l'encéphalopathie et l'accident vasculaire cérébral (AVC). ${ }^{1,2}$ Ces complications surviennent également dans d'autres contextes opératoires, ${ }^{3}$ mais l'incidence des complications après une chirurgie cardiaque est en général plus élevée que dans d'autres contextes. Dès lors, les patients en chirurgie cardiaque constituent une population qu'il est facile d'identifier pour l'étudier en détail. Aujourd'hui, il est plus que jamais pertinent de chercher à comprendre les facteurs influençant le devenir cérébral périopératoire; en effet, les patients se présentant pour subir une chirurgie sont non-seulement de plus en plus âgés (ce qui s'inscrit dans la lignée des changements démographiques mondiaux), mais ils présentent également souvent plusieurs comorbidités (dont plusieurs sont des facteurs de risque de lésion neurologique). Malgré les changements apportés à la prise en charge des maladies coronariennes qui ont provoqué un déclin progressif dans le nombre de pontages aortocoronariens (PAC) ces 5-10 dernières années, ces derniers demeurent l'une des opérations les plus pratiquées. De plus, selon une étude à grande échelle récente, le PAC constitue la méthode de prise en charge optimale des maladies pluritronculaires, contrairement aux interventions percutanées. ${ }^{4}$ Ainsi, nous pourrions assister à une recrudescence des chirurgies de PAC, ce qui provoquerait une augmentation encore plus importante du nombre global de patients manifestant un dysfonctionnement neurologique.

Il existe une littérature abondante concernant les facteurs de risque favorisant les complications neurologiques. ${ }^{5,6}$ Bien que nombre de ces facteurs soient modifiables, comme par exemple notre manière d'aborder les maladies athéromateuses de l'aorte ascendante, ${ }^{7}$ plusieurs ne le sont pas - ce qui est clairement le cas quand il est question de la composition génétique d'un individu. Plusieurs études récentes ont mis en exergue la manière dont notre environnement génétique peut soit augmenter notre prédisposition aux lésions neurologiques ou affecter notre capacité de récupération une fois qu'une telle lésion s'est produite. ${ }^{8-13}$ En dépit du fait qu'elles indiquent des facteurs de risque génétiques identifiables quoique parfois divergents, chacune de ces études comporte ses propres forces et faiblesses. Cependant, il faut garder à l'esprit que l'étude et la compréhension de la génomique périopératoire en sont à leurs balbutiements, et ce, indépendamment des comptes-rendus de découvertes considérables qui offrent des perspectives scientifiques novatrices. En effet, plusieurs faiblesses thématiques sont apparentes dans la plupart des études rapportées à ce sujet. Le premier et principal défaut de la plupart de ces études est lié à leur taille d'échantillon relativement petite. Bien que certaines de ces études soient plus vastes que la majorité des études cliniques périopératoires, elles ne le sont néanmoins pas suffisamment pour se soustraire aux problèmes de puissance statistique faible, de comparaisons multiples, et du domaine en constante évolution de la bio-informatique. Toutefois, les progrès des techniques analytiques grâce à des capacités et une précision étendues ont facilité la collecte des informations génétiques requises de façon plus rentable, ce qui permet désormais d'étudier des populations plus vastes.

La complexité incroyable de notre code génétique ${ }^{14}$ est un défi croissant qui se pose face à notre capacité de comprendre pleinement les effets des variations génétiques. Les variations dans la constitution génétique ont été décrites avec le concept de polymorphismes nucléotidiques (SNP), selon lequel les nucléotides (c.-à-d. les paires de bases) sont différents au sein de gènes spécifiques, ce qui produit des allèles différents. Les limites des techniques analytiques ont cependant restreint le nombre de SNP pouvant être raisonnablement interrogés. Cela nous a également menés à examiner des cibles spécifiques et prédéterminées, bien que biologiquement plausibles. Toutefois, ces SNP définis a priori pourraient passer complètement à côté des variantes plus importantes mais encore inconnues. Par conséquent, l'analyse du génotype complet, quoique présentant ses propres limites, a amélioré notre capacité de voir au delà des limites des variantes $a$ priori. ${ }^{15}$ Mais cette technique a également été décriée comme favorisant des recherches empiriques diffuses et sans hypothèse de base.

Gardant ces faits à l'esprit, il existe tout de même plusieurs études importantes et intrigantes, dans les domaines cardiaque et non cardiaque, qui ont permis d'étendre notre compréhension des devenirs neurologiques et de la génomique périopératoire. ${ }^{13,16}$ Les premières études portant sur les variations génétiques périopératoires et le devenir cérébral (il y a près de 15 ans) se sont concentrées sur les variantes d'un seul gène. Dans une étude de petite portée $(n=65)$ rapportée par Tardiff et coll., l'un des premiers SNP examinés était une variante de l'apolipoprotéine (APOE) dans laquelle l'allèle $\varepsilon 4$ était associé à un 
dysfonctionnement cognitif (DCPO) ${ }^{17}$ Les raisons qui ont poussé à examiner cette variante proviennent d'un corpus de littérature qui relatait certaines associations entre l'APOE et plusieurs complications cérébrales telles que la maladie d'Alzheimer, l'hémorragie sous-arachnoïdienne, le traumatisme crânien fermé ou d'autres. ${ }^{18,19}$ Toutefois, alors que plusieurs tentatives étaient menées par le biais de diverses études de corroboration, plusieurs questions de taille sont apparues quant au bien-fondé de la relation entre cette variante $\varepsilon 4$ et le DCPO. Par exemple, Steed et coll. n'ont pas pu démontrer de relation semblable dans un contexte de chirurgie cardiaque, et Gaynor et coll. ont plus tard découvert un résultat opposé dans une population de patients pédiatriques, où la variante $\varepsilon 2$ (et non pas $\varepsilon 4$ ) a été associée à des complications neurologiques. ${ }^{9,11}$ Dès lors, bien que Leung et coll. aient montré un lien potentiel entre l'APOE et le DCPO dans les cas de chirurgie non cardiaque ${ }^{20}$ relation également observée par Heyer et coll. chez les patients subissant une endartériectomie carotidienne, ${ }^{21}$ il est évident qu'il existe des variantes tout aussi importantes, si ce n'est plus.

Le deuxième SNP a être étudié en chirurgie cardiaque a été le polymorphisme vers le récepteur plaquettaire des intégrines $\left(\mathrm{PL}^{\mathrm{A} 2}\right)$. Mathew et coll. ont décrit l'impact de cette variation du constituant GPIIIa du récepteur plaquettaire de l'intégrine GPIIb/IIIa (relation étudiée en raison d'une association connue à une incidence accrue de syndromes coronariens aigus) ${ }^{22}$ sur le devenir des patients tels que déterminé à l'aide du MMSE (mini-mental status examination). ${ }^{10}$

Bien que cette étude $(n=70)$ ait démontré que les patients positifs au $\mathrm{PL}^{\mathrm{A} 2}$ obtenaient des résultats plus bas à l'examen MMSE, l'insensibilité relative des résultats du MMSE, de même que la taille réduite de l'échantillon à l'étude, ont quelque peu limité la portée des résultats.

Malgré les limites inhérentes à ces premières études, elles ont néanmoins établi des bases importantes pour la réalisation d'études postérieures plus élaborées associant génétique et complications cérébrales après une chirurgie cardiaque. Plus récemment, Grocott et coll. ont rapporté les résultats d'une étude de grande taille $(n=2104)$ examinant les liens entre les AVC survenant après une chirurgie cardiaque et jusqu'à 26 SNP différents. Cette étude a non seulement examiné un nombre bien plus élevé de SNP que jamais auparavant, mais elle a également tenu compte des interactions entre SNP. Par exemple, l'interaction entre les SNP de protéine C réactive (CRP) et d'interleukine 6 (IL6) a été associée à un risque significativement accru d'AVC. La combinaison de deux allèles mineurs de CRP (3'UTR $1846 \mathrm{C} / \mathrm{T})$ et IL-6 $(-174 \mathrm{G} / \mathrm{C})$ a triplé le risque d'AVC (rapport de cotes [RC], 3,3; intervalle de confiance [IC] $95 \%, 1,4-8,1 ; P=0,0023) .{ }^{23}$ L'incidence d'AVC dans une population possédant ce génotype était de 3,09\% par rapport à une incidence de $0,95 \%$ dans une population ne le possédant pas. Cette étude a mis en évidence une voie mécaniste potentielle de survenue de l'AVC périopératoire; les gènes inflammatoires ont supplanté les gènes liés à la thrombose dans leur relation avec l'AVC après une chirurgie cardiaque.

Lorsque des modalités d'imagerie et des examens neurologiques peuvent être utilisés afin d'identifier les déficits, l'AVC est, de plusieurs façons, plus facile à étudier dans un contexte périopératoire que le DCPO. Le phénotype du DCPO est un critère d'évaluation bien plus difficile à étudier car il nécessite des évaluations à la fois fastidieuses et détaillées de la fonction cognitive, lesquelles sont difficiles à réaliser à une échelle suffisamment importante pour comprendre pleinement les influences des variations génétiques. Malgré ces obstacles, Mathew et coll. ont décrit une étude sophistiquée $(n=443)$ présentant un effet nouveau de la sélectine P (SELP) et des SNP de protéine réactive $C .{ }^{8}$ Dans cette étude, les auteurs ont découvert que les allèles mineurs (mineurs en raison de leur survenue moins fréquente) CRP 1059G/C (RC 0,37, IC $95 \%: 0,16$ à 0,$78 ; P=0,013)$ et SELP $1087 \mathrm{G} / \mathrm{A}$ (RC 0,51 , IC $95 \%$ : $0,30$ à 0,$85 ; P=0,011)$ étaient en fait associés à une réduction du total des déficits cognitifs. La réduction de risque absolu d'incidence de DCPO était de 20,6 \% pour les patients possédant l'allèle CRP 1059C et de 15,2\% pour ceux possédant l'allèle SELP 1087A. En outre, les niveaux sériques périopératoires de CRP et les marqueurs de l'activation plaquettaire étaient également significativement plus bas chez les patients possédant une copie de ces allèles mineurs, ce qui appuie la plausibilité biologique d'une réduction de l'inflammation et de l'activation plaquettaire en présence des associations entre allèles observées ici, résultant en un devenir cognitif plus favorable.

Il ne fait aucun doute que le domaine de la génomique périopératoire est dynamique et en constante évolution. Tout comme notre compréhension première du DCPO et de sa relation aux lésions cérébrales a été supplantée par une compréhension de plus en plus complexe mais plausible d'un point de vue mécaniste, il faut s'attendre à ce qu'une compréhension plus sophistiquée des influences génétiques aille de pair avec le progrès des recherches dans ce domaine.

Et maintenant, où allons-nous? En premier lieu, en raison des limites statistiques et techniques décrites ci-dessus, tous les résultats significatifs (ceux déjà publiés ou à venir) doivent être corroborés. Il est difficile de savoir si les facteurs de risque génétiques déjà identifiés résisteront à un examen approfondi, mais cette phase est cruciale avant de passer à l'étape suivante - la stratification ciblée des risques génétiques. Jusqu'à ce que ces étapes intermédiaires soient mieux comprises, une étape encore plus lointaine qui 
va au delà de la compréhension des risques consiste en l'élaboration de plusieurs stratégies thérapeutiques selon la composition génétique d'un individu. Plusieurs briques ont été posées à ce jour, mais les fondations ne sont pas terminées. Les travaux en génomique périopératoire devraient à l'avenir permettre une meilleure compréhension de la physiopathologie, de l'évaluation des risques et, finalement, favoriser des tentatives de minimiser les effets des lésions neurologiques périopératoires grâce à une intervention préventive et thérapeutique.

Conflicts of interest None declared.

\section{References}

1. Newman MF, Kirchner JL, Phillips-Bute B, et al. Longitudinal assessment of neurocognitive function after coronary-artery bypass surgery. N Engl J Med 2001; 344: 395-402.

2. Roach GW, Kanchuger M, Mangano CM, et al. Adverse cerebral outcomes after coronary bypass surgery. Multicenter Study of Perioperative Ischemia Research Group and the Ischemia Research and Education Foundation Investigators. N Engl J Med 1996; 335: 1857-63.

3. Monk TG, Weldon BC, Garvan CW, et al. Predictors of cognitive dysfunction after major noncardiac surgery. Anesthesiology 2008; 108: 18-30.

4. Serruys PW, Morice MC, Kappetein AP, et al. Percutaneous coronary intervention versus coronary-artery bypass grafting for severe coronary artery disease. N Engl J Med 2009; 360: 961-72.

5. Arrowsmith JE, Grocott HP, Reves JG, Newman MF. Central nervous system complications of cardiac surgery. Br J Anaesth 2000; 84: 378-93.

6. Bucerius J, Gummert JF, Borger MA, et al. Stroke after cardiac surgery: a risk factor analysis of 16,184 consecutive adult patients. Ann Thorac Surg 2003; 75: 472-8.

7. Hammon JW, Stump DA, Butterworth JF, et al. Single crossclamp improves 6-month cognitive outcome in high-risk coronary bypass patients: the effect of reduced aortic manipulation. $\mathrm{J}$ Thorac Cardiovasc Surg 2006; 131: 114-21.

8. Mathew JP, Podgoreanu MV, Grocott HP, et al. Genetic variants in P-selectin and C-reactive protein influence susceptibility to cognitive decline after cardiac surgery. J Am Coll Cardiol 2007; 49: $1934-42$.
9. Gaynor JW, Gerdes M, Zackai EH, et al. Apolipoprotein E genotype and neurodevelopmental sequelae of infant cardiac surgery. J Thorac Cardiovasc Surg 2003; 126: 1736-45.

10. Mathew JP, Rinder CS, Howe JG, et al. Platelet PlA2 polymorphism enhances risk of neurocognitive decline after cardiopulmonary bypass. Multicenter Study of Perioperative Ischemia (McSPI) Research Group. Ann Thorac Surg 2001; 71: 663-6.

11. Steed L, Kong R, Stygall J, et al. The role of apolipoprotein E in cognitive decline after cardiac operation. Ann Thorac Surg 2001; 71: 823-6.

12. Tardiff BE, Newman $M F$, Saunders $A M$, et al. Preliminary report of a genetic basis for cognitive decline after cardiac operations. Ann Thorac Surg 1997; 64: 715-20.

13. Grocott HP. Genetic influences on cerebral outcome after cardiac surgery. Semin Cardiothorac Vasc Anesth 2006; 10: 291-6.

14. Venter JC, Adams MD, Myers EW, et al. The sequence of the human genome. Science 2001; 291: 1304-51.

15. Ikram MA, Seshadri S, Bis JC, et al. Genomewide Association Studies of Stroke. N Engl J Med 2009; 360: 1718-28.

16. Grocott HP. Genetics and outcome after cardiac surgery. J Extra Corpor Technol 2006; 38: 49-51.

17. Tardiff BE, Newman $M F$, Saunders $A M$, et al. Preliminary report of a genetic basis for cognitive decline after cardiac operations. The Neurologic Outcome Research Group of the Duke Heart Center. Ann Thorac Surg 1997; 64: 715-20.

18. Laskowitz DT, Vitek MP. Apolipoprotein E and neurological disease: therapeutic potential and pharmacogenomic interactions. Pharmacogenomics 2007; 8: 959-69.

19. Wilson M, Montgomery $H$. Impact of genetic factors on outcome from brain injury. Br J Anaesth 2007; 99: 43-8.

20. Leung JM, Sands LP, Wang Y, et al. Apolipoprotein E e4 allele increases the risk of early postoperative delirium in older patients undergoing noncardiac surgery. Anesthesiology 2007; 107: 40611.

21. Heyer EJ, Wilson DA, Sahlein DH, et al. APOE-epsilon4 predisposes to cognitive dysfunction following uncomplicated carotid endarterectomy. Neurology 2005; 65: 1759-63.

22. Zotz RB, Winkelmann BR, Nauck $M$, et al. Polymorphism of platelet membrane glycoprotein IIIa: human platelet antigen $1 \mathrm{~b}$ (HPA-1b/PlA2) is an inherited risk factor for premature myocardial infarction in coronary artery disease. Thromb Haemost 1998; 79: 731-5.

23. Grocott HP, White WD, Morris $R W$, et al. Genetic polymorphisms and the risk of stroke after cardiac surgery. Stroke 2005; 36: $1854-8$. 